\title{
A Bordism Viewpoint of Fiberwise Intersections
}

\author{
Gun Sunyeekhan \\ 50 Department of Mathematics, Faculty of Science, Kasetsart University, Bangkok 10900, Thailand
}

Correspondence should be addressed to Gun Sunyeekhan; fscigsy@ku.ac.th

Received 1 March 2013; Accepted 3 June 2013

Academic Editor: Nawab Hussain

Copyright (C) 2013 Gun Sunyeekhan. This is an open access article distributed under the Creative Commons Attribution License, which permits unrestricted use, distribution, and reproduction in any medium, provided the original work is properly cited.

We use the geometric data to define a bordism invariant for the fiberwise intersection theory. Under some certain conditions, this invariant is an obstruction for the theory. Moreover, we prove the converse of fiberwise Lefschetz fixed point theorem.

\section{Introduction}

In topological fixed point theory, there is a classical questions that people may ask; given a smooth self-map $f: M \rightarrow M$ of a smooth compact manifold $M$, when is $f$ homotopic to a fixed point free map?

The famous theorem, Lefschetz fixed point theorem, gave a sufficient condition to answer the above question as follows.

Theorem 1 (Lefschetz fixed point theorem). Let $f: M \rightarrow M$ be a smooth self-map of a compact smooth manifold $M$.

If $f$ has no fixed point, then the Lefschetz number $L(f)=0$, where $L(f)=\sum_{k \geq 0}(-1)^{k} \operatorname{Tr}\left(f_{* \mid} H_{k}(M ; \mathbb{Q})\right)$.

In general, the converse of the above theorem does not hold. It requires a more refined invariant than the Lefschetz number to make the converse hold (see [1-3]).

For this work, we focus on the similar arguments as above for the family of smooth maps over a compact base space $B$. The proof of the main theorem depends heavily on the intersection problem as follows.

From now on, the notations $X^{a}$ means the smooth manifold $X$ of dimension $a$ and $I$ means the unit interval $[0,1]$. If $Y^{b}$ is a submanifold of $X^{a}, f: X \rightarrow Z$, and $\eta$ is a bundle over $Z$, then $\nu_{Y \subseteq X}$ means the normal bundle of $Y$ in $X$ and $f^{*}(\eta)$ is a pull-back bundle of $\eta$ along the map $f$.

Later we define "framed bordism with coefficient in a bundle" as follows. Let $X$ be a smooth manifold with a bundle $\xi$ over it. Define $\Omega_{*}^{f r}(X ; \xi)$ to be the bordism groups of manifolds mapping to $X$, together with a stable isomorphism of the normal bundle with the pullback of $\xi$. This framed bordism group will be a home for our invariants $L^{\text {bord }}(f)$ (described in the last section) which detects more fixed point information than the regular Lefschetz number.

(I) Suppose that $E_{P}^{p+k}, E_{Q}^{q+k}$, and $E_{M}^{m+k}$ are smooth fiber bundles over a compact manifold $B^{k}$. Let $f: E_{P}^{p+k} \rightarrow E_{M}^{m+k}$ be a bundle map, and let $E_{Q}$ be a subbundle of $E_{M}$ with the inclusion bundle map $i_{Q}: E_{Q} \hookrightarrow E_{M}$.

We have a commutative diagram

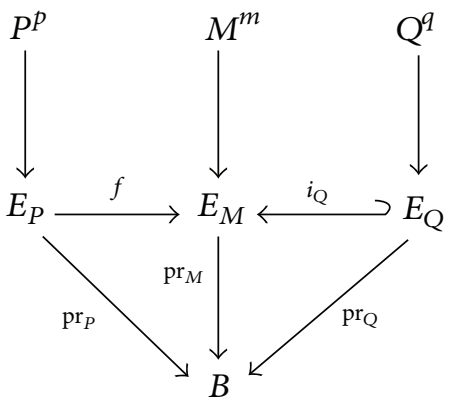

where $P, Q$, and $M$ are the fibers of $\operatorname{pr}_{P}, \operatorname{pr}_{Q}$, and $\mathrm{pr}_{M}$, respectively.

We may assume that $f \pitchfork E_{\mathrm{Q}}$ in $E_{M}$ (see [4]).

The homotopy pullback is

$$
\begin{aligned}
& E\left(f, i_{\mathrm{Q}}\right) \\
& \quad:=\left\{(x, \lambda, y) \in E_{P} \times E_{M}^{I} \times E_{\mathrm{Q}} \mid \lambda(0)=f(x), \lambda(1)=y\right\} .
\end{aligned}
$$


We have a diagram which commutes up to homotopy

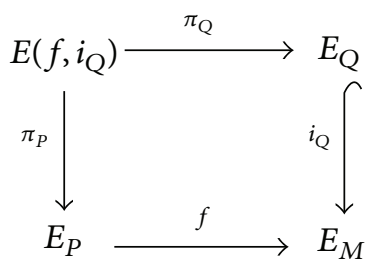

where $\pi_{P}$ and $\pi_{Q}$ are the trivial projections; that is, we have a homotopy $E\left(f, i_{\mathrm{Q}}\right) \times I \stackrel{K}{\rightarrow} E_{M}$ defined by $K(x, \lambda, y, t)=\lambda(t)$, $t \in I$.

We also have a map $c: f^{-1}\left(E_{\mathrm{Q}}\right) \rightarrow E\left(f, i_{\mathrm{Q}}\right)$ defined by $x \mapsto\left(x, c_{f(x)}, f(x)\right)$ where $c_{f(x)}=$ constant path in $E_{M}$ at $f(x)$.

Transversality yields a bundle map

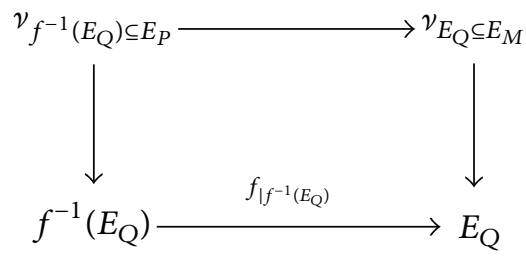

Choose an embedding $E_{P}^{p+k} \subseteq S^{L}$, for sufficiently large $L$, where $S^{L}$ is a sphere of dimension $L$. Then, we have $f^{-1}\left(E_{\mathrm{Q}}\right) \stackrel{i}{\hookrightarrow} E_{P}^{p+k} \subseteq S^{L}$. So $v_{f^{-1}\left(E_{\mathrm{Q}}\right) \subseteq S^{L}} \cong v_{f^{-1}\left(E_{\mathrm{Q}}\right) \subseteq E_{P}} \oplus i^{*} v_{E_{P} \subseteq S^{L}}$.

The commutative diagram

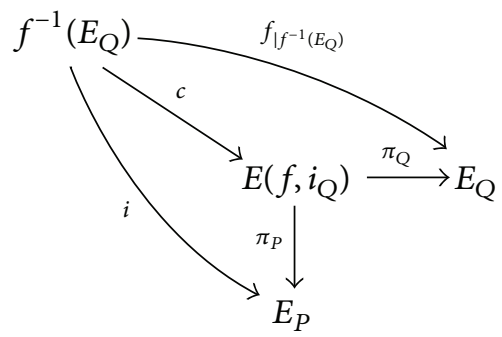

yields a bundle map

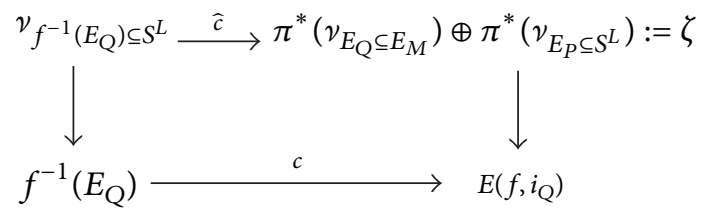

Thus, $(c, \widehat{c})$ determines an element $[c, \widehat{c}] \in \Omega_{p+q+k-m}^{f r}$ $\left(E\left(f, i_{\mathrm{Q}}\right) ; \xi\right)$.

(II) Suppose that $\left(N \stackrel{c_{1}}{\rightarrow} E\left(f, i_{\mathrm{Q}}\right), \nu_{N \subseteq S^{L}} \stackrel{\widehat{c}_{1}}{\rightarrow} \xi\right)$ is another representative of $[c, \widehat{c}]$, where $N^{p+q+k-m} \subseteq E_{P}^{p+k} \subseteq S^{L}$. This means we have a normal bordism $\left(W \stackrel{\mathscr{C}}{\rightarrow} E\left(f, i_{\mathrm{Q}}\right), \nu_{W} \stackrel{\widehat{\mathscr{C}}}{\rightarrow} \xi\right)$ between $(c, \widehat{c})$ and $\left(c_{1}, \widehat{c}_{1}\right)$; that is

(i) $W^{p+q+k-m+1} \subseteq\left(S^{L} \times I\right)$, (ii) $\partial W \subseteq\left(S^{L} \times \partial I\right)$,

(iii) $W \pitchfork\left(S^{L} \times \partial I\right)$,

(iv) $W \cap\left(S^{L} \times 0\right)=f^{-1}\left(E_{\mathrm{Q}}\right)$ and $W \cap\left(S^{L} \times 1\right)=N$

such that

$$
\begin{gathered}
\mathscr{C}_{\mid f^{-1}\left(E_{\mathrm{Q}}\right)}=c: f^{-1}\left(E_{\mathrm{Q}}\right) \longrightarrow E\left(f, i_{\mathrm{Q}}\right), \\
\mathscr{C}_{\mid N}=c_{1}: N \longrightarrow E\left(f, i_{\mathrm{Q}}\right), \\
\widehat{\mathscr{C}}_{\mid v_{f^{-1}\left(E_{\mathrm{Q}}\right) \subseteq S^{L}}}=\widehat{c}, \quad \widehat{\mathscr{C}}_{\mid v_{N \subseteq S^{L}}}=\widehat{c}_{1} .
\end{gathered}
$$

Theorem 2 (main theorem). Assume $m>q+((p+k) / 2)+1$. Then, there exists a smooth fiber-preserving map over $B$

$$
\Psi: E_{P} \times I \longrightarrow E_{M}
$$

such that $\Psi_{\mid E_{P} \times\{0\}}=f, \Psi \pitchfork E_{\mathrm{Q}}$ and $\Psi_{\mid E_{P} \times\{1\}}^{-1}\left(E_{\mathrm{Q}}\right)=N$.

Note that if we let $g=\Psi_{\mid E_{P} \times\{1\}}$, then $g$ is fiber-preserving homotopic to $f$ and $g^{-1}\left(E_{\mathrm{Q}}\right)=N$.

In 1974, Hatcher and Quinn [5] showed an interesting result as follows.

Theorem 3 (Hatcher-Quinn). Given smooth bundle maps $f$ : $E_{P} \rightarrow E_{M}$ and $i_{Q}: E_{Q} \rightarrow E_{M}$ over a compact base space $B$ which are immersions in each fiber, assume $m>q+((p+$ $k) / 2)+1$ and $m>p+((q+k) / 2)+1$. Then there is a fiberpreserving map $g: E_{P} \rightarrow E_{M}$ over B homotopic to $f$ such that $g^{-1}\left(E_{\mathrm{Q}}\right)=N$.

Their theorem required a map $f$ to be an immersion on each fiber which cannot be applied to our main theorem. We decided to give an alternative technique to proof the main theorem by constructing the required homotopy step by step. This techniques could be used as a model to achieve the similar result for the equivariant setting (see [6]).

\section{Proof of the Main Theorem}

Theorem 4 (Whitney embedding theorem). Let $M^{m}$ and $N^{n}$ be smooth manifolds, and let $f: M \rightarrow N$ be a smooth map. If $n>2 m$, then $f$ is homotopic to an embedding $g: M \rightarrow N$.

Lemma 5. Let $f: M^{m} \rightarrow N^{n}$ be a map between two smooth manifolds. Let $A$ be a closed submanifold of $M$. Assume that $f_{\mid A}$ is an embedding.

If $n>2 m$, then $f$ is homotopic to an embedding $g$ relative to $A$.

Proof. Let $T$ be a tubular neighborhood of $A$ in $M$.

Step 1. Extend the embedding $f_{\mid A}: A \rightarrow N$ to an embedding $f_{T}: T \rightarrow N$.

Let $v_{A \subseteq M}$ be the normal bundle of $A$ in $M$ and $D(v)$ denote the disc bundle of $\nu$. Then the tubular neighborhood theorem implies $D\left(v_{A \subseteq M}\right) \cong T$. 
Claim. For any given embedding $A \stackrel{g}{\hookrightarrow} N$ and vector bundle $\eta$ over $A$, then

( $g$ extends to an embedding of $D(\eta)$ into $N$ )

$\Longleftrightarrow$ (there exists a bundle monomorphism

$$
\phi: \eta \longrightarrow v(g))
$$

where $v(g)$ is the normal bundle of $A$ in $N$ via $g$.

Proof of Claim. $(\Leftarrow)$ We have a diagram

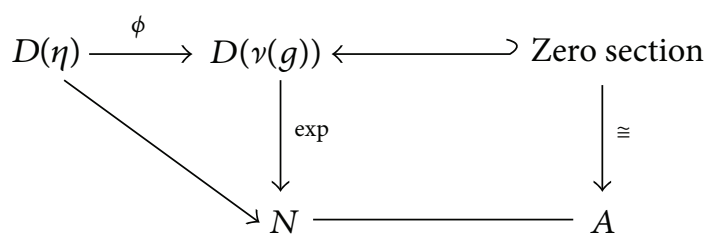

where exp is the exponential map.

Note that $\exp (D(\nu(g)) \cong$ tubular neighborhood of $A$ in $N$ via $g$. Then $\exp \circ \phi: D(\eta) \hookrightarrow N$ is a desired embedding.

$(\Rightarrow)$ Assume there exists an embedding $g_{T}$ so that the following diagram commutes

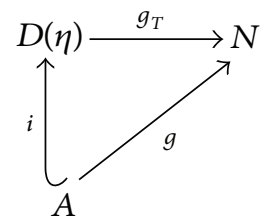

Then $v(g) \cong \eta \oplus i^{*} v\left(g_{T}\right)$.

We are in the situation where we have a commutative diagram

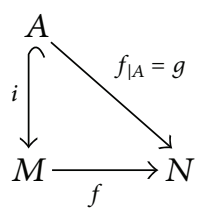

Let $v(f):=f^{*} \tau_{N}-\tau_{M}$. Then $i^{*}(\nu(f)) \oplus v_{A \subseteq M} \stackrel{\text { stable }}{\cong} v(g)$, where $\stackrel{\text { stable }}{\cong}$ denotes the stable isomorphism between 2 vector bundles.

If $n-a>a$, then $i^{*}(\nu(f)) \oplus v_{A \subseteq M} \cong \nu(g)$, so there exists a bundle monomorphism

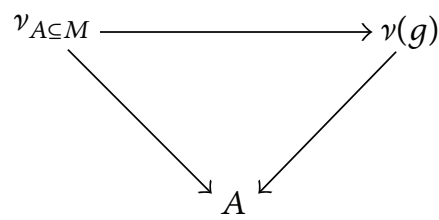

Apply the Claim when $g=f_{\mid A}$ and $\eta=v_{A \subseteq M}$. Then we have an extension embedding of $g$ from $D\left(\nu_{A \subseteq M}\right) \cong T \stackrel{f_{T}}{\longrightarrow} N$.
Step 2. We have a map $f_{\mid M-\operatorname{int} T}: M \backslash \operatorname{int}(T) \rightarrow N$ and $\partial(M \backslash$ $\operatorname{int}(T))=\partial T$.

The condition $b>(c+a / 2+1)$ and Theorem 4 imply that $f_{\mid M-\operatorname{int}(T)}$ is homotopic to an embedding $g_{M-\operatorname{int}(T)}$.

Define $g: M \rightarrow N$ by

$$
g(x)= \begin{cases}g_{M-\operatorname{int}(T)}(x) & \text { if } x \in M \backslash \operatorname{int}(T), \\ g_{T}(x) & \text { if } x \in T .\end{cases}
$$

Then $f$ is homotopic to $g$ relative to $A$.

2.1. Proof of the Main Theorem. We divide the proof into 3 steps.

Step 1. Goal: fiber-preserving homotoped the map $W \stackrel{a:=\pi_{P} \circ \mathscr{C}}{\longrightarrow}$ $E_{P}$ to an embedding over $B$.

By assumption, we have

$$
W \stackrel{\mathscr{C}}{\longrightarrow} E\left(f, i_{\mathrm{Q}}\right) \subseteq E_{P} \times E_{M}^{I} \times E_{\mathrm{Q}},
$$

and we also have maps

$$
W \stackrel{a:=\pi_{P} \circ \mathscr{C}}{\longrightarrow} E_{P}, \quad W \stackrel{b:=\pi_{Q^{\circ} \mathscr{C}}^{\longrightarrow}}{\longrightarrow} E_{\mathrm{Q}}, \quad W \times I \stackrel{H}{\longrightarrow} E_{M},
$$

where $H:=K \circ\left(\mathscr{C} \times \mathrm{id}_{I}\right)$, so $H_{\mid W \times 0}=f \circ a, H_{\mid W \times 1}=b$.

Recall that $\partial W=f^{-1}\left(E_{\mathrm{Q}}\right) \sqcup N, a_{\mid \partial W}$ is just the inclusion of $f^{-1}\left(E_{\mathrm{Q}}\right)$ and $N$ into $E_{P}$.

Apply the condition $m>q+((p+k) / 2)+1$ to Lemma 5, there exists an embedding $\mathscr{A} \simeq a(\operatorname{rel} \partial W)$. That is, we have a commutative diagram

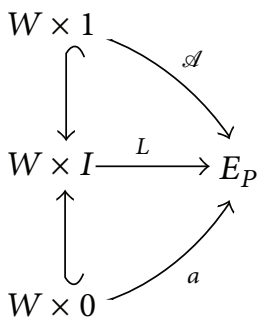

We have a map $W \stackrel{b:=\pi_{Q^{\circ \mathscr{C}}}}{\longrightarrow} E_{\mathrm{Q}}$. By concatenating the homotopy $H$ and $f \circ L$ together, we have a homotopy $V$ : $W \times[1,2] \rightarrow E_{M}$ such that $V(\rightarrow, 1)=\mathscr{A}$ and $V\left({ }_{-}, 2\right)=b$. Hence, $i_{Q} \circ b$ is homotopic to $f \circ \mathscr{A}$.

Next, we want to modify the homotopy $V$ such that it is fiber preserving with respect to $\mathrm{pr}_{M}$.

Note that we have a commutative diagram

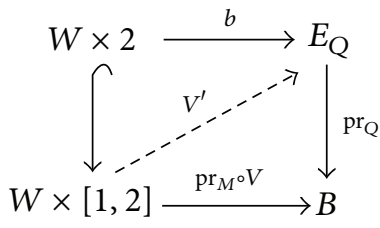


We can apply the homotopy lifting property for $\mathrm{pr}_{\mathrm{Q}}$ to get a homotopy of $b$ to $b^{\prime}$ through $V^{\prime}$ such that the following diagram commute:

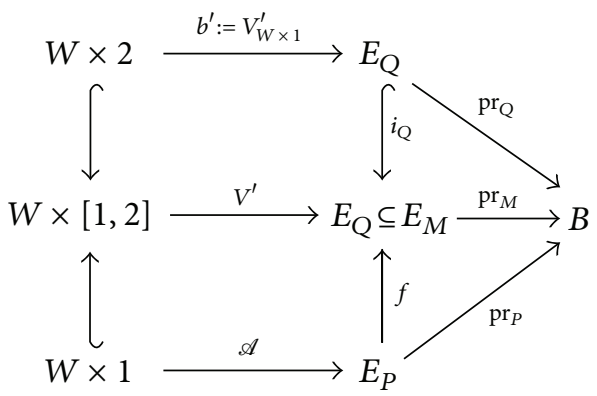

Let $\Psi_{W}:=b^{\prime}: W \rightarrow E_{\mathrm{Q}}$. Then $\Psi_{W}$ is a fiber-preserving map over $B$ through the lifting $V^{\prime}$.

Step 2. Goal: construct a bundle isomorphism

$$
\nu(\mathscr{A}) \oplus \epsilon^{1} \cong b^{\prime *}\left(\nu_{E_{Q} \subseteq E_{M}}\right),
$$

where $\epsilon^{1}$ is the trivial bundle.

Since $\operatorname{dim} W<\operatorname{rank} \nu(\mathscr{A})$, it is enough to give a stable equivalence between such bundles.

Now, we have

$$
W \stackrel{\mathscr{A}}{\hookrightarrow} E_{P} \subseteq S^{L} \Longrightarrow v_{W \subseteq S^{L}} \cong \nu(\mathscr{A}) \oplus \mathscr{A}^{*}\left(\nu_{E_{P} \subseteq S^{L}}\right) .
$$

We also have a commutative diagram

$$
\mathscr{A}^{\prime} \simeq a=\pi_{P} \circ \mathscr{C} \Longrightarrow \mathscr{A}^{*}\left(\nu_{E_{P} \subseteq S^{L}}\right) \cong\left(\pi_{P} \circ \mathscr{C}\right)^{*}\left(\nu_{E_{P} \subseteq S^{L}}\right),
$$

Thus, the bundle map $\widehat{\mathscr{C}}: \nu_{W \subseteq S^{L} \times I} \rightarrow \xi=\pi_{P}^{*}\left(\nu_{E_{P} \subseteq S^{L}}\right) \oplus$ $\pi_{\mathrm{Q}}^{*}\left(\nu_{E_{\mathrm{Q}} \subseteq E_{M}}\right)$ yields the following stable isomorphism

$$
\nu_{W \subseteq S^{L}} \oplus \epsilon \stackrel{\text { stable }}{\cong}\left(\pi_{P} \circ \mathscr{C}\right)^{*}\left(\nu_{E_{P} \subseteq S^{L}}\right) \oplus\left(\pi_{Q} \circ \mathscr{C}\right)^{*}\left(\nu_{E_{Q} \subseteq E_{M}}\right) .
$$

Putting (21), (23), and (24) together, we get

$$
\begin{aligned}
\nu(\mathscr{A}) \oplus\left(\pi_{P} \circ \mathscr{C}\right)^{*}\left(\nu_{E_{P} \subseteq S^{L}}\right) \oplus \epsilon^{1} \stackrel{\text { stable }}{\cong} & \left(\pi_{P} \circ \mathscr{C}\right)^{*}\left(\nu_{E_{P} \subseteq S^{L}}\right) \\
& \oplus b^{*}\left(\nu_{E_{Q} \subseteq E_{M}}\right) .
\end{aligned}
$$

Consequently, we have

$$
\nu(\mathscr{A}) \oplus \epsilon^{1} \cong b^{\prime *}\left(\nu_{E_{Q} \subseteq E_{M}}\right) .
$$

This implies that we did construct a bundle map

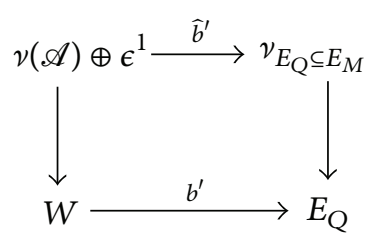

which gives us the extension of map $b^{\prime}$ to the tubular neighborhood of $W$ in $E_{P}$. More precisely,

$$
\Psi_{T}: D(\nu(\mathscr{A})) \hookrightarrow D\left(\nu(\mathscr{A}) \oplus \epsilon^{1}\right) \stackrel{\widehat{b^{\prime}}}{\longrightarrow} D\left(v_{E_{Q} \subseteq E_{M}}\right),
$$

where $D$ denotes the disc bundle. Note that $\Psi_{T} \pitchfork E_{Q}$ and $\Psi_{T}\left(\partial D\left(\eta_{1}\right)\right) \subseteq E_{M} \backslash E_{\mathrm{Q}}$.

Since $\nu(\mathscr{A}) \oplus \epsilon^{1} \cong b^{\prime *}\left(\nu_{E_{Q} \subseteq E_{M}}\right)$, we can find a subbundle $\eta_{2}$ of $b^{\prime *}\left(\nu_{E_{Q} \subseteq E_{M}}\right)$ such that $\eta_{2} \cong \nu(\mathscr{A})$. For simplicity, let $\eta_{1}:=$ $\nu(\mathscr{A})$.

Step 3. Goal: construct the fiber-preserving smooth map $\Psi$ : $E_{P} \times I \rightarrow E_{M}$ over $B$.

Recall that we have

$$
W \hookrightarrow D(\nu(\mathscr{A})) \simeq D\left(\nu(\mathscr{A}) \oplus \epsilon^{1}\right) \cong D\left(b^{\prime *}\left(\nu_{E_{\mathrm{Q}} \subseteq E_{M}}\right)\right) .
$$

Then there exists a neighborhood $\bar{D}$ of $W$ in $D\left(b^{\prime *}\right.$ $\left.\left(\nu_{E_{Q} \subseteq E_{M}}\right)\right)$ such that $\bar{D} \simeq D\left(b^{\prime *}\left(\nu_{E_{Q} \subseteq E_{M}}\right)\right)$ and $\bar{D} \cong D(\nu(\mathscr{A}))$.

According to (19), we have a commutative diagram

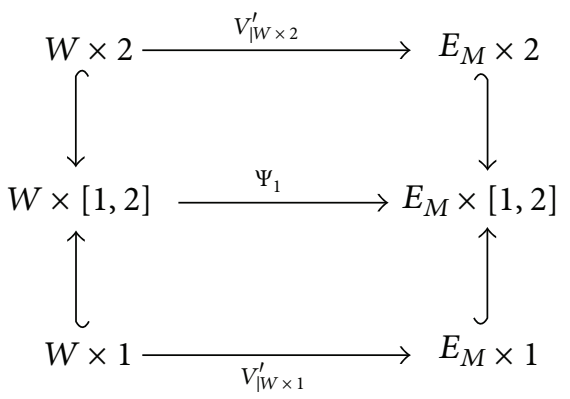

where $\Psi_{1}(w, t)=\left(V^{\prime}(w, t), t\right)$.

Let $\eta_{1}=v\left(\mathscr{A} \oplus \epsilon^{1}\right), \eta_{2}=b^{*}\left(\nu_{E_{Q} \subseteq E_{M}}\right)$.

According to (27), there exists a bundle $\eta$ over $W \times I$ such that $\eta_{\mid W \times i}=\eta_{i}$ for $i=1,2$.

Let $D_{1}:=D(\nu(\mathscr{A})), D_{2}:=\bar{D}$. Then $D_{i} \hookrightarrow D\left(\eta_{i}\right)$ is a homotopy equivalence for $i=1,2$ and also $D_{1} \cong D_{2}$.

Since $D_{1} \cup W \times[1,2] \cup D_{2} \hookrightarrow D_{1} \times[1,2]$ is a cofibration and a homotopy equivalence, there exist an extension 
$D_{1} \times[1,2] \stackrel{\widehat{\Psi}_{1}}{\longrightarrow} E_{M} \times[1,2]$ such that the following diagram commutes

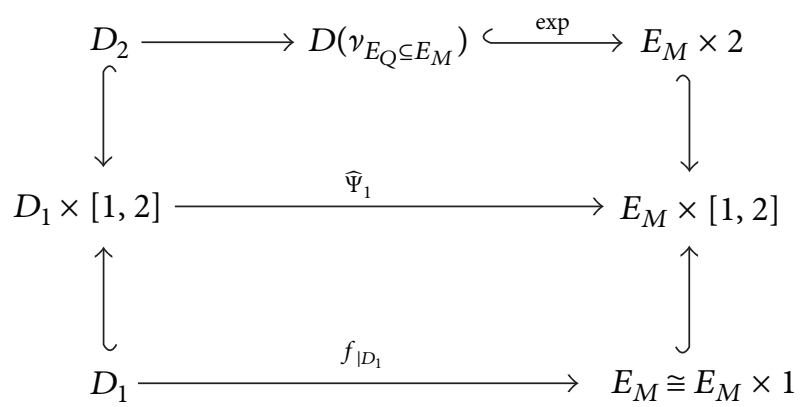

Next we want to construct an embedding $W \stackrel{\mathscr{A}^{\prime}}{\hookrightarrow} D_{2} \times[2,3]$ such that the following hold:

(i) $\mathscr{A}^{\prime}(W) \cap\left\{D_{2} \times 2\right\}=f^{-1}\left(E_{\mathrm{Q}}\right)$,

(ii) $\mathscr{A}^{\prime}(W) \cap\left\{D_{2} \times 3\right\}=N$,

(iii) $\mathscr{A}^{\prime} \pitchfork D_{2} \times \partial[2,3]$.

We start by letting $\alpha: W \rightarrow[2,3]$ be a smooth map such that $\alpha \pitchfork \partial[2,3], \alpha^{-1}(2)=f^{-1}\left(E_{Q}\right)$ and $\alpha^{-1}(3)=N$, and we also have an inclusion $W \stackrel{i_{W}}{\hookrightarrow} D_{2}$.

Let $\mathscr{A}^{\prime}:=i_{W} \times \alpha$. Then $\mathscr{A}^{\prime}$ is such a required map.

By the construction, we have

$$
D\left(\nu\left(\mathscr{A}^{\prime}\right)\right) \cong D_{2} \times[2,3]
$$

Let $\psi_{2}$ be the composition of the maps

$$
W \stackrel{\mathscr{A}^{\prime}}{\longrightarrow} D_{2} \times[2,3] \stackrel{\Psi_{T \mid D_{2}} \times \operatorname{id}_{[2,3]}}{\longrightarrow} M \times[2,3] \stackrel{\text { proj }}{\longrightarrow} M
$$

Define a map $\Psi_{2}:=\psi_{2} \times \alpha: W \rightarrow E_{M} \times[2,3]$.

Using the fact that $D\left(\nu\left(\mathscr{A}^{\prime}\right)\right) \cong D_{2} \times[2,3]$, then $D_{2} \times$ $\partial[2,3] \cup \mathscr{A}^{\prime}(W) \hookrightarrow D_{2} \times[2,3]$ is a cofibration and homotopy equivalence. Hence there exists an extension $D_{2} \times[2,3] \stackrel{\widehat{\Psi}_{2}}{\longrightarrow}$ $E_{M} \times[2,3]$.

Note that for $(x, 3) \in D_{2} \times 3$ such that $\widehat{\Psi}_{2}(x, 3) \in E_{Q} \times 3$, the map $\widehat{\Psi}_{2 \mid D_{2} \times 3}=\Psi_{T}$ forces that $x$ has to be in $W$, so the definition of $\Psi_{2}$ implies $x \in N$. Thus

$$
\widehat{\Psi}_{2 \mid D_{2} \times 3}^{-1}\left(E_{\mathrm{Q}}\right)=N \text {. }
$$

We define a map $\widetilde{\Psi}:\left\{E_{P} \times I\right\} \cup\left\{D_{1} \times[1,2]\right\} \cup\left\{D_{2} \times[2,3]\right\} \rightarrow$ $E_{M} \times[0,3]$ by

$$
\widetilde{\Psi}(p, t)= \begin{cases}(f(p), t) & \text { if } t \in[0,1] \\ \left(\widehat{\Psi}_{1}(p), t\right) & \text { if } t \in[1,2] \\ \left(\widehat{\Psi}_{2}(p), t\right) & \text { if } t \in[2,3]\end{cases}
$$

Then $\widetilde{\Psi}$ is well-defined map over $B$ by the construction.
It's not hard to see that $\left\{E_{P} \times I\right\} \cup\left\{D_{1} \times[1,2]\right\} \cup\left\{D_{2} \times\right.$ $[2,3]\}$ is diffeomorphic to $E_{P} \times I$. Define the map $\Psi$ to be the composition of maps

$$
\begin{aligned}
E_{P} \times I & \stackrel{\cong}{\longrightarrow}\left\{E_{P} \times I\right\} \cup\left\{D_{1} \times[1,2]\right\} \cup\left\{D_{2} \times[2,3]\right\} \\
& \stackrel{\widetilde{\Psi}}{\longrightarrow} E_{M} \times[0,3] \stackrel{\text { proj }}{\longrightarrow} E_{M},
\end{aligned}
$$

where proj is the projection to the first factor.

Thus, we get a map $\Psi: E_{P} \times I \rightarrow E_{M}$ over $B$ so that $\Psi_{\mid E_{P} \times 0}=f$. By construction, $\Psi \pitchfork E_{Q}$ and $\Psi_{\mid E_{P} \times 1}^{-1}\left(E_{Q}\right)=N$ as required.

Corollary 6. Assume $m>q+((p+k) / 2)+1$. Then the map $f$ can be fiber-preserving homotoped to a map whose image does not intersect $E_{Q}$ if and only if $[c, \widehat{c}]=0 \in$ $\Omega_{p+q+k-m}^{f r}\left(E\left(f, i_{\mathrm{Q}}\right) ; \xi\right)$.

\section{Application to Fixed Point Theory}

Let $p: M^{m+k} \rightarrow B^{k}$ be a smooth fiber bundle with compact fibers and $k>2$. Assume that $B$ is a closed manifold. Let $f$ : $M \rightarrow M$ be a smooth map over $B$. That is, $p \circ f=p$.

The fixed point set of $f$ is

$$
\operatorname{Fix}(f):=\{x \in M \mid f(x)=x\} .
$$

We have a homotopy pull-back diagram

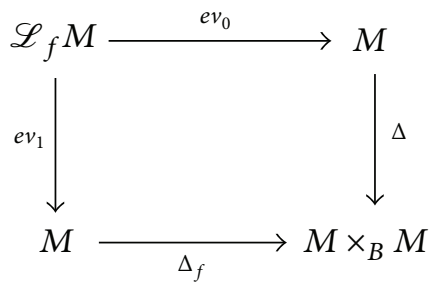

where

(i) $\mathscr{L}_{f} M:=\left\{\alpha \in M^{I} \mid f(\alpha(0))=\alpha(1)\right\}$,

(ii) $e v_{0}$ and $e v_{1}$ are the evaluation map at 0 and 1 respectively,

(iii) $\Delta:=$ the diagonal map, defined by $x \mapsto(x, x)$,

(iv) $\Delta_{f}:=$ the twisted diagonal map, defined by $x \mapsto$ $(x, f(x))$,

(v) $M \times{ }_{B} M:=$ the fiber bundle over $B$ with fiber over $b \in$ $B$, given by $F_{b} \times F_{b}$ where $F_{b}$ is the fiber of $p$ over $b$.

Proposition 7. There exists a homotopy from $f$ to $f_{1}$ such that $\Delta_{f_{1}} \pitchfork \Delta$.

The proof relies on the work of Koźniewski [4], relating to $B$-manifolds. Let $B$ be a smooth manifold. A $B$-manifold is a manifold $X$ together with a locally trivial submersion $p$ : $X \rightarrow B$. A $B$-map is a smooth fiber-preserving map.

Lemma 8. Let $X$ and $Y$ be B-manifolds, and let $Z$ be a $B$ submanifold of $Y$. Let $g: X \rightarrow Y$ be a B-map. Then, there 
is a fiber-preserving smooth B-homotopy $H_{t}: X \rightarrow Y$ such that $H_{0}=g$ and $H_{1} \pitchfork Z$.

Proof. See [7] for the proof.

We have a transversal (pull-back) square

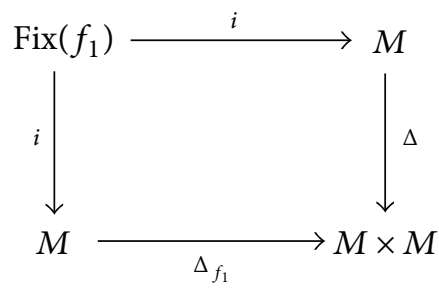

where $i$ is the inclusion. Transversality yields that $v(i) \cong$ $i^{*}(\nu(\Delta)) \cong i^{*}(\tau M)$.

Choose an embedding $M \hookrightarrow S^{m+k}$ for sufficiently large $k$. Then we have

$$
\begin{aligned}
\nu_{\mathrm{Fix}\left(f_{1}\right) \subseteq S^{m+k}} & \cong \nu(i) \oplus i^{*}\left(\nu_{M \subseteq S^{m+k}}\right) \\
& \cong i^{*}(\tau M) \oplus i^{*}\left(\nu_{M \subseteq S^{p+k}}\right) \cong \epsilon .
\end{aligned}
$$

We denote this bundle isomorphism by $\nu_{\mathrm{Fix}\left(f_{1}\right) \subseteq S^{m+k}} \stackrel{\widehat{g}}{\rightarrow} \epsilon$. We also have a map $\operatorname{Fix}\left(f_{1}\right) \stackrel{g}{\rightarrow} \mathscr{L}_{f} M$ defined by $x \mapsto c_{x}$, where $c_{x}$ is the constant map at $x$. Thus $L^{\text {bord }}(f):=\left[\operatorname{Fix}\left(f_{1}\right), g, \widehat{g}\right]$ determines the element in $\Omega_{k}^{f r}\left(\mathscr{L}_{f} M ; \epsilon\right)$.

Applying Theorem 2, We obtain the following corollary.

Corollary 9 (converse of fiberwise Lefschetz fixed point theorem). Let $f: M^{m+k} \rightarrow M^{m+k}$ be a smooth bundle map over the closed manifold $B^{k}$. Assume that $m \geq k+3$. Then, $f$ is fiber homotopic to a fixed point free map if and only if $L^{\text {bord }}(f)=0 \in \Omega_{k}^{f r}\left(\mathscr{L}_{f} M ; \epsilon\right)$.

Corollary 10. Let $f: M^{m+k} \rightarrow M^{m+k}$ be a smooth bundle map over the closed manifold $B^{k}$. Assume that $m \geq k+3$. If there is $\left[N, N \stackrel{g}{\rightarrow} \mathscr{L}_{f} M, v_{N \subseteq S^{m+k}} \stackrel{\widehat{g}}{\rightarrow} \epsilon\right] \in \Omega_{k}^{f r}\left(\mathscr{L}_{f} M ; \epsilon\right) f$ where $N$ is a finite subset of $M$ such that $L^{\text {bord }}(f)=[N, g, \hat{g}]$, then the map $f$ can be fiber-preserving homotoped to a map $h$ such that $\operatorname{Fix}(h)=N$.

\section{Acknowledgments}

The author gratefully acknowledge the Coordinating Center for Thai Government Science and Technology Scholarship Students (CSTS) and the National Science and Technology Development Agency (NSTDA) for funding this research. The author would also like to thank the referees, P.M. Akhmet'ev Akhmet'ev and Hichem Ben-El-Mechaiekh, for all the comments and suggestions.

\section{References}

[1] F. Wecken, "Fixpunktklassen. I," Mathematische Annalen, vol. 117, pp. 659-671, 1941 (German).
[2] J. Nielsen, "Über die Minimalzahl der Fixpunkte bei den Abbildungstypen der Ringflächen," Mathematische Annalen, vol. 82, no. 1-2, pp. 83-93, 1920 (German).

[3] B. J. Jiang, "Fixed point classes from a differential viewpoint," in Fixed Point Theory (Sherbrooke, Que., 1980), vol. 886 of Lecture Notes in Mathematics, pp. 163-170, Springer, Berlin, Germany, 1981.

[4] T. Koźniewski, “The category of submersions," L’Académie Polonaise des Sciences. Bulletin. Série des Sciences Mathématiques, vol. 27, no. 3-4, pp. 321-326, 1979.

[5] A. Hatcher and F. Quinn, "Bordism invariants of intersections of submanifolds," Transactions of the American Mathematical Society, vol. 200, pp. 327-344, 1974.

[6] G. Sunyeekhan, Equivariant intersection theory [Ph.D. thesis], University of Notre Dame, Notre Dame, Indiana, 2010.

[7] V. Coufal, A family version of Lefschetz-Nielsen fixed point theory [Ph.D. thesis], University of Notre Dame, Notre Dame, Indiana, 2004. 


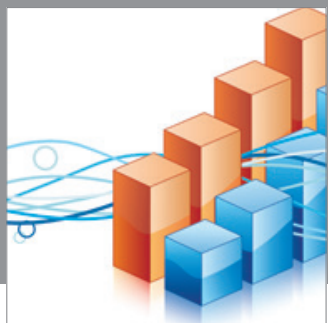

Advances in

Operations Research

mansans

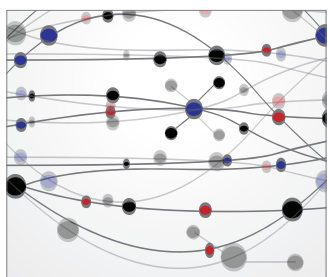

The Scientific World Journal
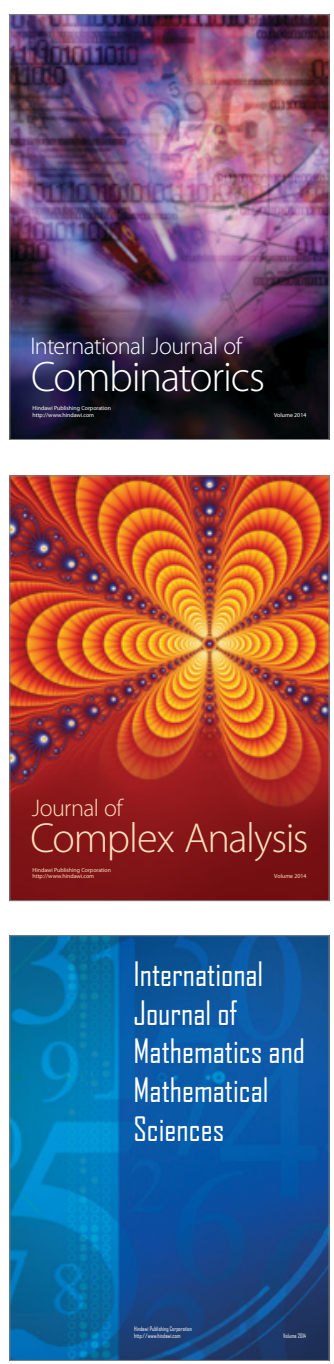
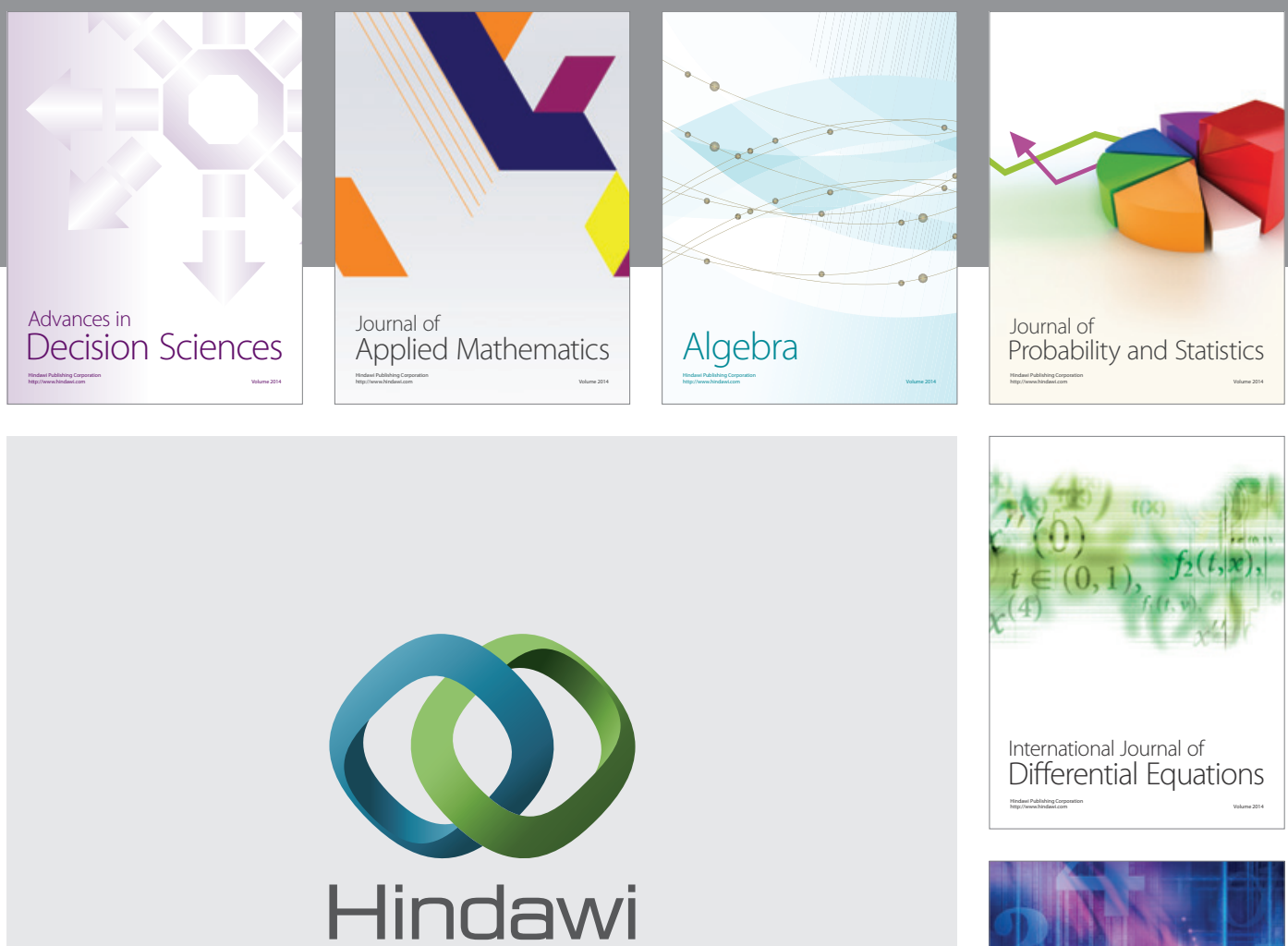

Submit your manuscripts at http://www.hindawi.com
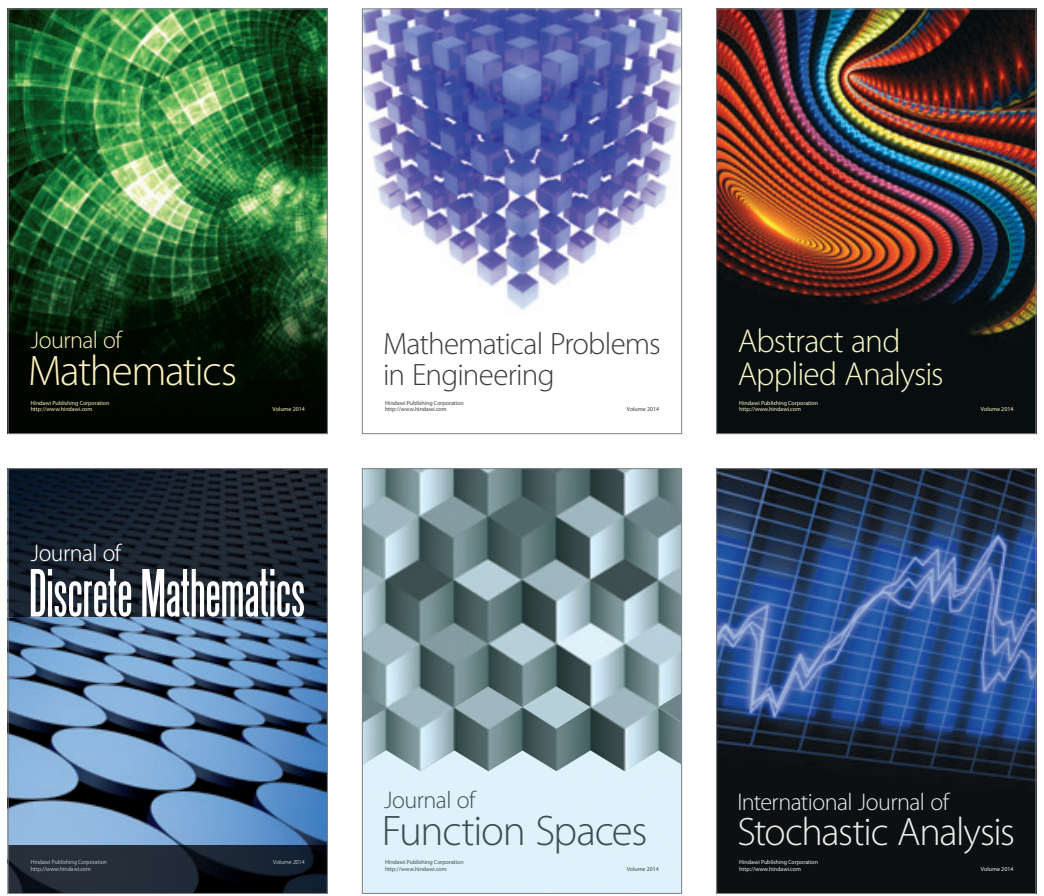

Journal of

Function Spaces

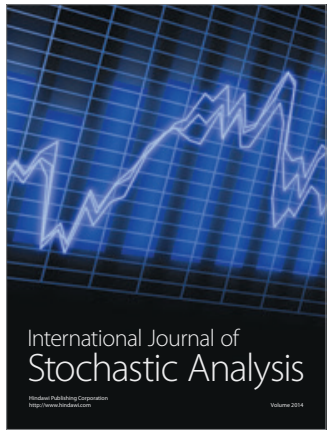

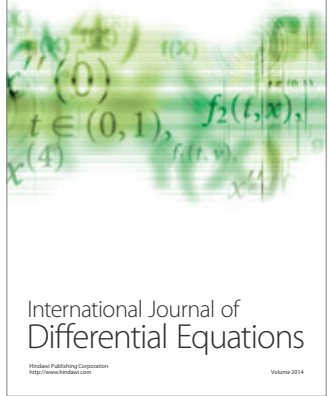
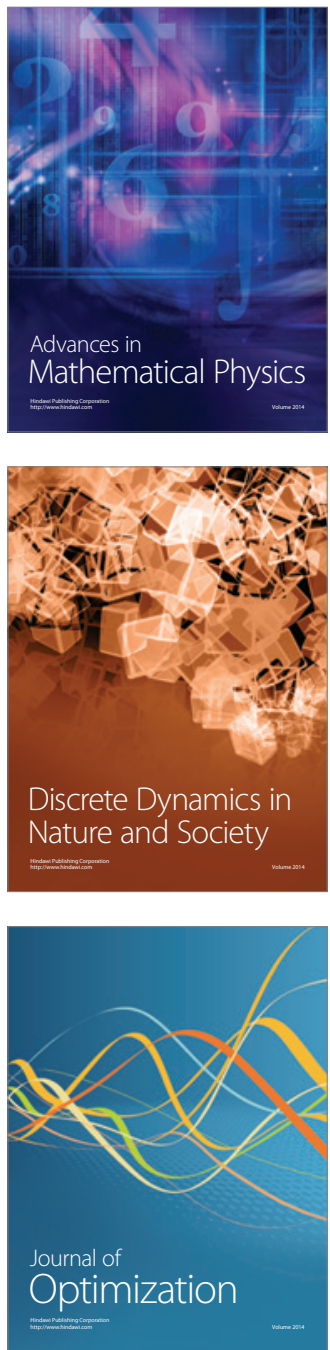